\title{
RESIDUOS DE PLAGUICIDAS EN SUELOS DE USO AGRÍCOLA Y RIESGO DE EXPOSICIÓN EN LA MICROCUENCA LOS ZARZALES, MUNICIPIO RIVAS DÁVILA, ESTADO MÉRIDA, VENEZUELA
}

(Pesticide residues in agricultural soils of Los Zarzales micro-watershed, Rivas Dávila municipality, Mérida state, Venezuela)

\author{
José Alberto ROJAS-FERNÁNDEZ ${ }^{1}$, Pedro Rafael BENÍTEZ-DÍAZ ${ }^{2}$, \\ Edgard Alejandro RIVAS-ROJAS ${ }^{1}$ y Leticia MIRANDA-CONTRERAS ${ }^{1 *}$
}

${ }^{1}$ Centro de Microscopía Electrónica, Universidad de Los Andes, Calle 32 entre Avenida 4 y Tulio Febres Cordero, Mérida 5101 - República Bolivariana de Venezuela

${ }^{2}$ Departamento de Botánica y Ciencias Básicas, Escuela de Ingeniería Forestal, Facultad de Ciencias Forestales y Ambientales, Universidad de Los Andes, Avenida Principal Chorros de Milla, Conjunto Forestal, Edificio Principal, 1er piso, Mérida 5101 - República Bolivariana de Venezuela

*Autor para correspondencia: lmiranda@ula.ve

(Recibido enero 2018; aceptado agosto 2018)

Palabras clave: carbendazim, carbofurano, mancozeb, paraquat, agroquímicos

\section{RESUMEN}

La preocupación sobre la presencia de residuos de plaguicidas en suelos de uso agrícola es cada vez mayor debido a que constituyen un riesgo ambiental y una fuente de exposición humana. En este trabajo se determinó la presencia de estos residuos y el riesgo de exposición en la microcuenca Los Zarzales, municipio Rivas Dávila, Mérida, Venezuela. Se tomaron muestras de suelos en la etapa inicial, media y final de cultivos de ciclo corto, se extrajeron los principios activos y se analizaron por cromatografía líquida de alta resolución. El riesgo de exposición se estimó calculando la dosis de plaguicidas a través de la ingesta no dietética de suelo y polvo contaminado, según los criterios de la US-EPA. La mayor concentración y variedad de compuestos se registró durante la etapa media: $26 \pm 7 \mathrm{mg} / \mathrm{kg}$ de residuos totales y $100 \%$ de frecuencia de detección, destacando entre ellos paraquat $(15 \pm 7 \mathrm{mg} / \mathrm{kg})$, carbendazim $(8 \pm 2 \mathrm{mg} / \mathrm{kg})$, mancozeb $(3.7 \pm 0.7 \mathrm{mg} / \mathrm{kg})$ y carbofurano $(1.2 \pm 0.5 \mathrm{mg} / \mathrm{kg})$. Con base en estos valores, las dosis diarias calculadas fueron: $1.5,0.8,0.37$ y $0.12 \mu \mathrm{g} /$ día, respectivamente, todas las cuales resultaron estar por debajo de la ingesta diaria admisible establecida por la Organización de las Naciones Unidas para la Agricultura y la Alimentación y la Organización Mundial de la Salud, que es utilizada como referencia. A pesar de ello, la dosis total alcanzó los $2.8 \mu \mathrm{g} /$ día, lo cual se considera que puede constituir un riesgo de exposición.

Key words: carbendazim, carbofuran, mancozeb, paraquat, agrochemicals

\begin{abstract}
There is a growing concern about the presence of pesticide residues in agricultural land because they constitute an environmental risk and a source of human exposure. In this work, the presence of these residues and the risk of exposure was determined in the
\end{abstract}


micro-watershed of Los Zarzales, Rivas Dávila municipality, Mérida, Venezuela. Soil samples were taken in the initial, middle and final stages of short cycle crops, and the active ingredients were extracted and analyzed by HPLC. The risk of exposure was estimated by calculating the dose of pesticides through non-dietary intake of contaminated soil and dust, according to the US-EPA criteria. The highest concentration and variety of compounds was recorded during the middle stage: $26 \pm 7 \mathrm{mg} / \mathrm{kg}$ of total residues and $100 \%$ detection frequency, standing out among them paraquat $(15 \pm 7 \mathrm{mg} / \mathrm{kg})$, carbendazim $(8 \pm 2 \mathrm{mg} / \mathrm{kg})$, mancozeb $(3.7 \pm 0.7 \mathrm{mg} / \mathrm{kg})$ and carbofuran $(1.2 \pm 0.5 \mathrm{mg} / \mathrm{kg})$. Based on these values, the calculated daily doses were $1.5,0.8,0.37$ and $0.12 \mu \mathrm{g} / \mathrm{day}$, respectively, all of which were below the admissible daily intake established by $\mathrm{FAO} /$ WHO, which is used as reference. Despite of this, the total dose reached $2.8 \mu \mathrm{g} /$ day, which is considered as a risk of exposure.

\section{INTRODUCCIÓN}

En Venezuela, al igual que en muchos otros países de Latinoamérica, se lleva a cabo una práctica agrícola altamente dependiente de la aplicación de productos químicos, destacándose entre éstos los plaguicidas sintéticos. Este grupo de compuestos juega un papel muy importante en el control de plagas y enfermedades que pueden perjudicar los cultivos, $\mathrm{y}$ actualmente su aplicación es fundamental para mantener los niveles de productividad y rentabilidad de la actividad agrícola. Sin embargo, su uso inadecuado implica una seria amenaza para los agricultores, los trabajadores que lo aplican, habitantes de las zonas de producción y consumidores finales de los productos cosechados, además de causar daños irreversibles al ambiente (Benítez-Díaz y Miranda-Contreras 2013). Más aún, estudios realizados en animales de experimentación y en cultivo de tejido neuronal, arrojan indicios de que la exposición a estas sustancias tóxicas puede afectar el desarrollo del sistema nervioso central (Benítez-Díaz y Miranda-Contreras 2009, Peña-Contreras et al. 2016).

Los plaguicidas que caen al suelo pueden ser transportados a lugares más allá de su sitio de aplicación. A través de los fenómenos de arrastre y lixiviación, sus residuos pueden contaminar cursos de aguas subterráneas y superficiales, alcanzando también los reservorios de agua para consumo humano que son alimentados por éstos (Vymazal y Brezinová 2015). Se ha encontrado evidencia de que el uso inadecuado de estas sustancias en zonas de intensa actividad agrícola, como es el caso del municipio Rivas Dávila del estado Mérida, Venezuela, ha provocado la contaminación de aguas superficiales y de consumo humano (Benítez-Díaz y Miranda-Contreras 2013). Se ha estudiado también la presencia de sus residuos en rubros de alto consumo producidos en esa zona, como papa (Solanum tuberosum L.) y fresa (Fragaria $x$ ananassa), en los cuales se encontraron cantidades alarmantemente altas de organofosforados, ditiocarbamatos y carbamatos, entre los cuales se detectó la presencia de algunos disruptores endocrinos como carbofurano, clorpirifos, diazinon, mancozeb y metomilo (Benítez-Díaz et al. 2015a, b). Más aún, la exposición ocupacional a este último conjunto de compuestos ha tenido un impacto negativo en la función reproductora masculina de los trabajadores agrícolas de la zona (Miranda-Contreras et al. 2013, 2015). La situación también parece estar afectando a niños en edad escolar que habitan en el municipio, en quienes se han reportado alteraciones hematológicas como trombocitosis y disminución de la capacidad antioxidante total (Miranda-Contreras et al. 2017).

Otro aspecto relevante vinculado con la presencia de residuos de plaguicidas en suelos de uso agrícola es el impacto de estas sustancias sobre los organismos no-blanco, particularmente en la microflora del suelo, cuyas funciones son críticas para la productividad y sostenibilidad de la actividad agrícola y la calidad del ambiente (Imfeld y Vuilleumier 2012). Adicionalmente, se ha reportado que la presencia de estos compuestos en polvo y suelo constituye una importante vía de exposición humana, ya que las partículas contaminadas pueden ser inhaladas y adherirse fácilmente a la piel, mucosas, alimentos y otros objetos, a través de los cuales pueden ser ingeridas por los agricultores y los habitantes de las zonas de producción y sus alrededores, siendo la población infantil la más susceptible debido a su tendencia a jugar en el suelo y llevarse objetos y manos a la boca (Simcox et al. 1995, US-EPA 2011).

El propósito de este trabajo fue determinar la presencia de residuos de plaguicidas en muestras de suelo obtenidas en una zona de intensa actividad agrícola, específicamente en la microcuenca Los Zarzales ubicada en el municipio Rivas Dávila del estado Mérida, Venezuela, así como estimar el riesgo de exposición para los trabajadores y habitantes de la zona. Los suelos estudiados se utilizan en siembras de 
ciclo corto y el muestreo se realizó en tres periodos del proceso de cultivo. Los principios activos analizados fueron: bipiridilo (paraquat), carbamatos (metomilo y carbofurano), ditiocarbamato (mancozeb), organofosforados (dimetoato, diazinon y profenofos), benzimidazol (carbendazim), piretroides (cialotrina y cipermetrina) y triazina (metribuzin).

\section{MATERIALES Y METODOS}

\section{Reactivos y estándares}

Los estándares de los plaguicidas metomilo (Mt), carbofurano $(\mathrm{Cb})$, dimetoato $(\mathrm{Dm})$, diazinon $(\mathrm{Dz})$, profenofos $(\mathrm{Pf})$, carbendazim $(\mathrm{Cz})$, cialotrina $(\mathrm{Cl})$, cipermetrina $(\mathrm{Cm})$ y metribuzin $(\mathrm{Mb})$ fueron obtenidos de la casa comercial AccuStandard; mancozeb (Mz) de Merck, y paraquat (Pq) de Sigma, todos con pureza superior al 98\%. Los disolventes orgánicos utilizados fueron de grado apto para cromatografía líquida de alta resolución (HPLC, por sus siglas en inglés): metanol y hexano de J.T. Baker, dicloroetano y acetona de Merck. El sulfato de sodio anhidro fue de grado analítico de Merck. El agua ultra pura fue obtenida de un sistema de purificación Millipore MilliQ.

\section{Área de estudio}

El área de estudio es la vertiente norte de la microcuenca Los Zarzales, que forma parte del municipio Rivas Dávila del estado Mérida, Venezuela. Geográficamente se ubica entre los $08^{\circ} 10^{\prime} 00^{\prime}$ " y $08^{\circ} 15^{\prime} 00^{\prime \prime}$ de latitud norte, y los $71^{\circ} 52^{\prime} 30^{\prime \prime}$ y $71^{\circ} 55^{\prime} 00^{\prime}$ " de longitud oeste. Tiene una superficie aproximada de $25.07 \mathrm{~km}^{2}$ y la superficie cultivada es de alrededor de $3.77 \mathrm{~km}^{2}$. Forma parte de la cuenca alta del río Mocotíes, que se ubica al suroeste del estado Mérida (Delgado et al. 1998, Benítez-Díaz y Miranda-Contreras 2013). La confluencia del río Los Zarzales con el río Las Tapias, $2.5 \mathrm{~km}$ aguas arriba de Bailadores, forman el río Mocotíes, el cual constituye el principal drenaje del municipio.

\section{Muestreo de suelos}

Se seleccionaron nueve parcelas con características agroecológicas similares, localizadas alrededor de $1800 \mathrm{msnm}$, representativas de la zona cultivada de la microcuenca, con una superficie mínima de 2500 y máxima de $15000 \mathrm{~m}^{2}$. En estas parcelas se realizan, de forma rotativa, cultivos de hortalizas de ciclo corto, entre los que se encuentran papa (Solanum tuberosum), zanahoria (Daucus carota) y cebollín (Allium schoenoprasum). Una vez seleccionadas las parcelas, se realizó una entrevista con los productores para identificar los plaguicidas más utilizados y su forma de aplicación. Las muestras se tomaron entre los meses de enero y abril de 2017, correspondientes al final del periodo seco e inicio del periodo lluvioso. El muestreo de suelo se llevó a cabo en tres etapas del cultivo: inicial (preparación del terreno), media (mitad del periodo de cultivo) y final (al momento de la cosecha). De acuerdo con procedimientos previamente descritos y validados en la literatura (Mejías y Jerez 2006, FHIA 2007, Uzcátegui et al. 2011), en cada etapa y parcela se tomó una muestra compuesta de $1 \mathrm{~kg}$ de suelo, la cual estaba constituida por la homogeneización de 10 submuestras tomadas en forma de zigzag, con la finalidad de cubrir la mayor cantidad de terreno posible. Al tomar las muestras se tuvo cuidado de que estuvieran libres de restos vegetales y piedras, utilizando una pala y cavando un hueco en forma de $\mathrm{V}$, a una profundidad de $30 \mathrm{~cm}$, para evitar el exceso de plaguicidas que eventualmente pudieran estar en la superficie del suelo. Luego se almacenaron en bolsas plásticas con cierre hermético y al llegar al laboratorio se mantuvieron refrigeradas a $4{ }^{\circ} \mathrm{C}$ hasta el momento de realizar los análisis. En cada etapa se tomaron un total de nueve muestras, una por cada parcela, las cuales fueron analizadas por triplicado.

\section{Análisis de residuos de plaguicidas}

Los plaguicidas analizados fueron escogidos de acuerdo con entrevistas realizadas en el campo y con apoyo de la literatura existente sobre estudios previamente realizados en la zona (Flores-García et al. 2011, Molina-Morales et al. 2012, BenítezDíaz y Miranda-Contreras 2013, Benítez-Díaz et al. 2015a, b).

Para la extracción de los residuos de plaguicidas se utilizó el método EPA 3540C (US-EPA 1996). En primer lugar se realizó un tratamiento previo que consistió en secar la muestra a temperatura ambiente para luego pulverizarla y tamizarla a través de un tambor de $1 \mathrm{~mm}$. Posteriormente se realizó la extracción mediante Soxhlet, para lo cual se mezclaron $10 \mathrm{~g}$ de la muestra con $10 \mathrm{~g}$ de $\mathrm{Na}_{2} \mathrm{SO}_{4}$ y se colocaron en el dedal de extracción. Luego se agregaron $300 \mathrm{~mL}$ de una mezcla 1:1 de hexano-acetona, en un matraz de fondo redondo de $500 \mathrm{~mL}$ acoplado al Soxhlet; la muestra se extrajo durante $24 \mathrm{~h}$ a una velocidad de 4 a 6 ciclos/h. Transcurrido este tiempo, el extracto se dejó enfriar y luego se concentró en un rotavapor a $110 \mathrm{rpm}$ y $95^{\circ} \mathrm{C}$; seguidamente se añadieron $5 \mathrm{~mL}$ de hexano. Posteriormente se evaporó el disolvente bajo atmósfera de nitrógeno para luego resuspender la muestra en $500 \mu \mathrm{L}$ de metanol y por último someterla al análisis por HPLC. 
Para el análisis de plaguicidas por HPLC-DAD, se empleó el método descrito por Flores-García et al. (2011) con un equipo de HPLC Agilent modelo 1200 constituido por una bomba binaria, desgasificador, inyector manual Rheodyne de $100 \mu \mathrm{L}$, compartimiento termostático para columna y detector de arreglo de diodos (DAD). Los parámetros del sistema y los datos cromatográficos fueron controlados y analizados usando el software ChemStation. Se utilizó una columna de fase reversa $\mathrm{C}_{18}$ Spherisorb de $5 \mu \mathrm{m}$ de tamaño de partícula y dimensiones de $4.6 \times 250 \mathrm{~mm}$. Para la separación se empleó un gradiente lineal de $0-100 \%$ de B en $115 \mathrm{~min}$, a un flujo de $0.7 \mathrm{~mL} / \mathrm{min}$, donde la fase móvil A fue metanol en agua (20\%), ajustada a pH 4.6 con ácido fosfórico, y la fase móvil $\mathrm{B}$, metanol en agua (90\%), pH 4.6. Los compuestos fueron identificados por su tiempo de retención y absorbancia a 220, 230 y $300 \mathrm{~nm}$. La cuantificación se realizó a través del método del estándar externo.

\section{Cálculo de la dosis diaria de plaguicidas a través de suelo contaminado}

A partir de las concentraciones de plaguicidas en suelo (Ps, $\mathrm{mg} / \mathrm{kg}$ ) se calculó la posible dosis diaria por grupo etario (DdPS, $\mu \mathrm{g} /$ día), utilizando como base las estimaciones hechas por la US-EPA (2011) para consumo de polvo y suelo (Cps, mg/día), las cuales son de $100 \mathrm{mg}$ /día para niños de 1 a 10 años, y 50 $\mathrm{mg} /$ día para adultos (20 años de edad), empleando la siguiente ecuación:

$$
D d P S=C p s \times P s \times 10^{-3}
$$

Este cálculo se hizo para los principios activos encontrados en mayor concentración y para su total en la etapa media del cultivo, por ser los niveles más elevados y los que conllevan mayor riesgo.

Con la finalidad de tener un término de comparación, se calculó la ingesta diaria admisible (IdA, $\mu \mathrm{g} /$ día) para cada uno de los plaguicidas encontrados en mayor concentración y para los tres grupos etarios. El cálculo se realizó a partir de la ingesta diaria admisible (ADI, por sus siglas en inglés, $\mu \mathrm{g} /$ día/kg peso corporal) establecida por la Organización de las Naciones Unidas para la Agricultura y la Alimentación (FAO) y la Organización Mundial de la Salud (OMS) (EUFIC 2013, EC 2016) y el peso corporal promedio (PC) para la población venezolana, publicado en los Archivos Latinoamericanos de Nutrición (Landaeta-Jiménez et al. 2013), utilizando la siguiente ecuación:

$I d A=A D I \times P C$

\section{Estadística}

Los resultados se presentan como el promedio más/menos la desviación estándar. La significación estadística se determinó a través de una prueba de análisis de varianza (ANDEVA), con el programa GraphPad InStat 4.

\section{RESULTADOS}

De acuerdo con la información suministrada por los agricultores, la cantidad de plaguicidas utilizados al inicio del cultivo es menor debido a que el requerimiento fundamental es aportar fertilizantes al terreno. A medida que se desarrolla el cultivo, se incrementa la cantidad y frecuencia de aplicación para mantener el producto sano. Cuando se acerca la cosecha, disminuye el número y volumen de principios activos aplicados para incrementar la utilización de productos como hormonas y fertilizantes sintéticos, que favorecen el aumento de tamaño de los rubros cultivados.

En el cuadro I se muestran los niveles de plaguicidas encontrados en suelos de uso agrícola de la microcuenca Los Zarzales. En el periodo inicial de los cultivos la frecuencia de detección fue aproximadamente del $73 \%$, incrementándose al $100 \%$ en la etapa media, para disminuir al $64 \%$ en la cosecha. Los principios activos $\mathrm{Cb}, \mathrm{Cl}, \mathrm{Cz}, \mathrm{Pq}, \mathrm{Mt}$, Dm y Pf fueron detectados en todos los muestreos; $\mathrm{Mz}$ se encontró en las etapas inicial y media; $\mathrm{Dz}, \mathrm{Cm}$ y $\mathrm{Mb}$ sólo se presentaron a la mitad del tiempo de cultivo.

En la figura 1 se muestra la variación de los niveles de los plaguicidas presentes en mayor concentración. En primer lugar está Pq, cuyos niveles no presentan diferencias estadísticamente significativas entre la primera $(9.1 \pm 0.7 \mathrm{mg} / \mathrm{kg})$ y segunda etapas $(15 \pm 7 \mathrm{mg} / \mathrm{kg}$ ), aunque se observa una tendencia al incremento para disminuir casi 34 veces $(0.48 \pm$ $0.09 \mathrm{mg} / \mathrm{kg}, \mathrm{p}<0.001)$ al momento de la cosecha. En segundo lugar, se encuentra $\mathrm{Cz}$, cuyos niveles se mantienen prácticamente constantes (alrededor de 7 $\mathrm{mg} / \mathrm{kg}$ ) durante el estudio, seguido por $\mathrm{Mz}$, que no cambia significativamente en los dos periodos en que fue detectado (3.6 y $3.7 \mathrm{mg} / \mathrm{kg}$ ). Por último, Cf se mantiene alrededor de $0.9 \mathrm{mg} / \mathrm{kg}$ desde la preparación del terreno hasta la cosecha, aunque tiene una ligera tendencia a disminuir en cada periodo.

En la figura 2 se muestra la variación de los niveles totales de plaguicidas. Se observa una tendencia al aumento entre la etapa inicial y media, a pesar de que la diferencia no es estadísticamente significativa. En el momento de la cosecha, la concentración total 
CUADRO I. RESIDUOS DE PLAGUICIDAS (mg/kg) EN SUELOS DE USO AGRÍCOLA UBICADOS EN LA MICROCUENCA LOS ZARZALES, PARROQUIA LAS PLAYITAS DEL MUNICIPIO RIVAS DÁVILA DEL ESTADO MÉRIDA, VENEZUELA

\begin{tabular}{|c|c|c|c|c|}
\hline \multirow{3}{*}{$\begin{array}{l}\text { Grupo Químico } \\
\text { Bipiridilo }\end{array}$} & \multirow{3}{*}{$\begin{array}{l}\text { Plaguicida } \\
\text { Paraquat }\end{array}$} & \multicolumn{3}{|c|}{ Etapa del cultivo } \\
\hline & & Inicio & Medio & Final \\
\hline & & $9.1 \pm 0.7$ & \pm 7 & $0.48 \pm 0.09$ \\
\hline Carbamato & $\begin{array}{l}\text { Metomilo } \\
\text { Carbofurano }\end{array}$ & $\begin{array}{l}0.027 \pm 0.007 \\
1.2 \pm 0.5\end{array}$ & $\begin{array}{l}0.04 \pm 0.01 \\
0.9 \pm 0.2\end{array}$ & $\begin{array}{l}0.019 \pm 0.005 \\
0.6 \pm 0.3\end{array}$ \\
\hline Ditiocarbamato & $\begin{array}{l}\text { Mancozeb } \\
\text { Dimetoato }\end{array}$ & $\begin{array}{ll}3.6 & \pm 0.4 \\
0.47 & \pm 0.06\end{array}$ & $\begin{array}{ll}3.7 & \pm 0.7 \\
0.53 & \pm 0.08\end{array}$ & $\begin{array}{c}\text { ND } \\
0.44 \stackrel{ \pm 0.09}{ }\end{array}$ \\
\hline Organofosforado & $\begin{array}{l}\text { Diazinon } \\
\text { Profenofos }\end{array}$ & $\begin{array}{l}\mathrm{ND} \\
0.06 \pm 0.02\end{array}$ & $\begin{array}{l}0.06 \pm 0.02 \\
0.11 \pm 0.02\end{array}$ & $\begin{array}{l}\text { ND } \\
0.05 \pm 0.04\end{array}$ \\
\hline Benzimidazol & Carbendazim & $8 \pm 2$ & $6 \pm 1$ & \pm 1 \\
\hline Piretroide & $\begin{array}{l}\text { Cialotrina } \\
\text { Cipermetrina }\end{array}$ & $\begin{array}{c}0.05 \pm 0.01 \\
\mathrm{ND}\end{array}$ & $\begin{array}{l}0.04 \pm 0.01 \\
0.031 \pm 0.009\end{array}$ & $\begin{array}{c}0.05 \pm 0.01 \\
\mathrm{ND}\end{array}$ \\
\hline Triazina & $\begin{array}{l}\text { Metribuzin } \\
\text { Total }\end{array}$ & $\begin{array}{l}\mathrm{ND} \\
\pm 2\end{array}$ & $\begin{aligned} 0.025 & \pm 0.004 \\
26 & \pm 7\end{aligned}$ & $\begin{array}{l}\text { ND } \\
\pm 1\end{array}$ \\
\hline
\end{tabular}

ND: no detectado

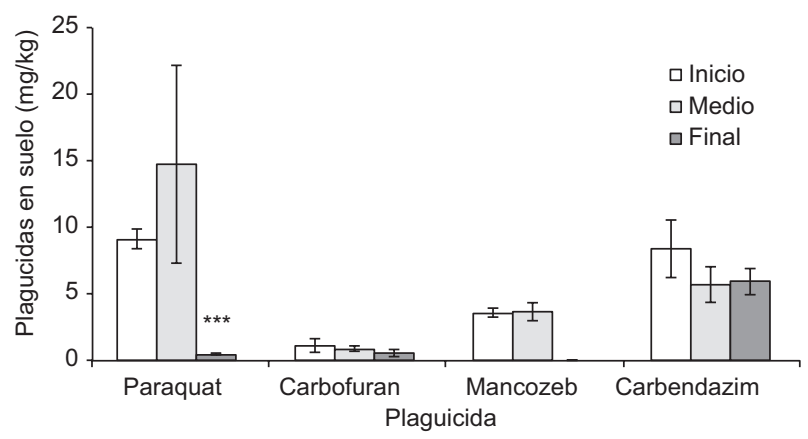

Fig. 1. Variación de los niveles de los plaguicidas presentes en mayor concentración en suelos de uso agrícola ubicados en la microcuenca Los Zarzales, parroquia Las Playitas del municipio Rivas Dávila del estado Mérida, Venezuela $(* * * \mathrm{p}<0.001 ; \mathrm{n}=9)$

es tres veces menor que en el periodo intermedio, siendo esta diferencia estadísticamente significativa.

En el cuadro II se presenta una comparación entre la IdA según la edad (1, 10 y 20 años) y la DdPS; como se indicó en la sección de materiales y métodos, el cálculo se realizó sobre los plaguicidas presentes en mayor concentración, los cuales se encontraron en la etapa media de cultivo, a excepción del $\mathrm{Cz}$, cuya mayor concentración se encontró en la etapa inicial. Se puede apreciar que de forma individual, en ninguno de los casos la DdPS sobrepasa el IdA, y que

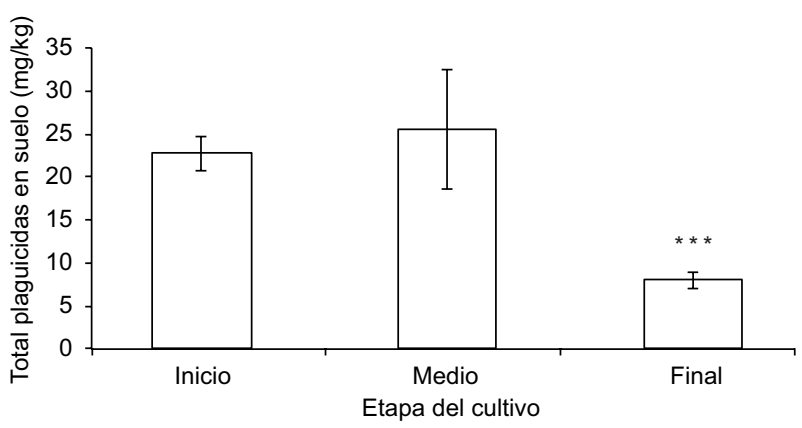

Fig. 2. Variación de las concentraciones totales de plaguicidas encontrados en suelos de uso agrícola ubicados en la microcuenca Los Zarzales, parroquia Las Playitas del municipio Rivas Dávila del estado Mérida, Venezuela $(* * * \mathrm{p}<0.001 ; \mathrm{n}=9)$

la cantidad total de plaguicidas que se podría ingerir por esta vía de exposición alcanza los $2.6 \mu \mathrm{g}$ diarios.

\section{DISCUSIÓN}

De acuerdo con los datos mostrados en el cuadro I, se puede identificar una tendencia general caracterizada por un incremento de la frecuencia de detección de plaguicidas entre la etapa inicial y media de los cultivos, y una disminución importante en la etapa 
CUADRO II. INGESTA DIARIA ADMISIBLE (mg/día) DE LOS PLAGUICIDAS ENCONTRADOS EN MAYOR CONCENTRACIÓN, CALCULADA A PARTIR DE LA INGESTA DIARIA ADMISIBLE (ADI, POR SUS SIGLAS EN INGLÉS) Y EL PESO CORPORAL (PC) PROMEDIO POR EDAD EN VENEZUELA (SE COMPARA CON LA DOSIS DIARIA DE PLAGUICIDA POR CONSUMO DE POLVO Y SUELO CONTAMINADO A PARTIR DE LOS NIVELES DE PLAGUICIDAS EN SUELO ENCONTRADOS EN LA ETAPA MEDIA DE LOS CULTIVOS)

\begin{tabular}{|c|c|c|c|c|c|c|}
\hline \multirow{2}{*}{ Plaguicida } & \multirow{2}{*}{$\begin{array}{c}\mathrm{ADI}^{*} \\
\mu \mathrm{g} / \mathrm{kg} / \mathrm{día}\end{array}$} & \multicolumn{3}{|c|}{$\begin{array}{l}\text { Ingesta diaria admisible por edad } \\
\text { (IdA, } \mu \mathrm{g} / \text { día) }\end{array}$} & \multicolumn{2}{|c|}{$\begin{array}{l}\text { Dosis diaria por polvo } \\
\text { y suelo (DdPS, } \mu \mathrm{g} / \text { día) }\end{array}$} \\
\hline & & $\begin{array}{l}\text { Edad 1año } \\
\text { PC } 9.6 \mathrm{~kg}\end{array}$ & $\begin{array}{l}\text { Edad } 10 \text { años } \\
\text { PC } 28.9 \mathrm{~kg}\end{array}$ & $\begin{array}{l}\text { Edad } 20 \text { años } \\
\text { PC } 60.8 \mathrm{~kg}\end{array}$ & $\begin{array}{l}\text { Edad } \\
1 \text { a } 10 \text { años }\end{array}$ & $\begin{array}{l}\text { Edad } \\
\text { Adulta }\end{array}$ \\
\hline $\mathrm{Pq}$ & 4 & 40 & 100 & 200 & 1.5 & 0.75 \\
\hline $\mathrm{CF}$ & 0.15 & 1 & 4 & 9 & 0.12 & 0.06 \\
\hline $\mathrm{Mz}$ & 50 & 500 & 1400 & 3040 & 0.37 & 0.19 \\
\hline $\mathrm{Cz}$ & 20 & 200 & 600 & 1200 & 0.8 & 0.4 \\
\hline Total & & & & & 2.6 & 1.3 \\
\hline
\end{tabular}

ADI: ingesta diaria admisible según FAO/OMS (EUFIC 2013). Las ADI mostradas en la tabla se obtuvieron de la EU Pesticides Database (EC 2016)

final. Este comportamiento también se observa en los niveles totales mostrados en la figura 2; aunque no es significativo, es evidente un incremento entre la etapa inicial y media, así como una disminución estadísticamente significativa en el último periodo. Los patrones observados coinciden con la práctica agrícola que se realiza en la zona, en la cual se utilizan agroquímicos desde la preparación del terreno para la siembra. Su uso se incrementa tanto en cantidad como en variedad a medida que se desarrollan los cultivos, para garantizar su éxito, y disminuye al acercarse la cosecha, cuando se debe suspender su aplicación para lograr que el producto cosechado esté apto para el consumo humano; en otras palabras, para lograr que los residuos de plaguicidas que pueden estar presentes en los alimentos alcancen concentraciones por debajo de los límites máximos de residuos (LMR) establecidos en el Codex Alimentarius (FAO/ OMS 2016). Los datos confirman lo reportado en trabajos anteriores, donde se indica que en la práctica agrícola del municipio Rivas Dávila se aplican los plaguicidas antes de que aparezca la plaga, de acuerdo con los paradigmas establecidos por la revolución verde iniciada en la década de 1950 (Benítez-Díaz y Miranda-Contreras 2013).

En general, se podría decir que los niveles de plaguicidas encontrados en las muestras de suelo son relativamente bajos, aunque sobresalen entre ellos Pq, Cf, Mz y Cz (Cuadro I, Fig. 1). Los tres primeros se apegan a la tendencia general identificada previamente, pero no sucede así con $\mathrm{Cz}$, que se mantiene prácticamente constante en las tres etapas. Este último es un fungicida sistémico de amplio espectro que se aplica antes, durante y después de la cosecha, y tiene una persistencia de dos a tres semanas, según su hoja técnica (Silvestre 2015). De acuerdo con evaluaciones realizadas por otros autores, la velocidad de degradación del $\mathrm{Cz}$ en el suelo se incrementa con aplicaciones sucesivas, disminuyendo su persistencia de 9.3 a 0.9 días después de cuatro aplicaciones (Yu et al. 2009). Por tanto, los resultados podrían indicar que este principio activo fue utilizado de forma poco frecuente pero en grandes cantidades, con el objetivo de mantener controlada la aparición de hongos hasta el momento de la cosecha. Este tipo de práctica podría traer como consecuencia el incremento de los niveles de residuos en los alimentos cosechados, ya que no se espera el tiempo requerido para que sean degradados en el ambiente o metabolizados por las plantas después de su última aplicación (Alister et al. 2017). En apoyo a esta observación, se ha reportado la presencia de residuos de organofosforados, ditiocarbamatos y carbamatos por encima de sus LMR en papa y fresa producidos en la zona (Benítez-Díaz et al. 2015a, b).

En Venezuela no existen regulaciones sobre los niveles de residuos de plaguicidas en suelo y los organismos multinacionales como OMS, FAO o la Unión Europea tampoco lo han establecido, por lo que es difícil establecer la forma en que estos residuos afectan la salud y calidad de vida de los trabajadores y habitantes de las zonas de producción agrícola. Dentro de este contexto, algunos autores han sugerido que la ingesta de polvo y suelo contaminado podría constituir un importante medio de exposición (Simcox et al. 1995, Morgan et al. 2014, Richards et al. 2016). Esta ingesta no dietética afectaría en mayor grado a los grupos etarios más bajos, es decir, niños en edad preescolar y escolar, debido a su mayor 
tendencia a jugar en el suelo y a llevarse las manos y otros objetos a la boca. Como término de referencia para la estimación del riesgo de exposición, se utilizan los ADI que comúnmente se emplean para los alimentos, en el entendido de que se refieren a la dosis diaria de una sustancia química que ingerida durante todo el periodo vital, parece no entrañar riesgo apreciable para la salud del consumidor, sobre la base de todos los hechos conocidos en el momento de la evaluación de la sustancia química por la Reunión Conjunta FAO/OMS sobre residuos de plaguicidas (EUFIC 2013).

Los resultados obtenidos (Cuadro II) parecen indicar que esta vía de exposición no conlleva un riesgo apreciable. Sin embargo, se deben destacar dos aspectos importantes: en primer lugar, en un trabajo publicado por Morgan et al. (2014) se consideraron los riesgos de exposición a plaguicidas en niños que habitaban zonas urbanas a través de tres vías combinadas que incluían la dietética, no dietética (polvo y suelo) e inhalación. Se encontró que la ingesta total varía de 1.71 a $12.5 \mathrm{ng} / \mathrm{kg} /$ día, lo cual está dos a tres órdenes de magnitud por debajo de lo reportado en el presente trabajo; a pesar de ello, los autores consideraron que estos niños tenían un importante riesgo de exposición.

En segundo lugar, en la mayoría de los casos, si no en todos, cuando se hace este tipo de estudio se considera cada principio activo por separado; es decir, como si se analizaran casos aislados de contaminación por cada uno de ellos, cuando en realidad habría que considerar la exposición simultánea a diferentes compuestos que podrían actuar de manera aditiva o sinérgica. Así, la mezcla de activos podría ser potencialmente más tóxica que cada uno por separado, incluso cuando sus niveles individuales se encuentren por debajo de la dosis consideradas tóxicas (Alam y Jones 2014, Ferré et al. 2018, García-Hernández et al. 2018). A pesar de esto, ninguna agencia de protección ambiental ha establecido un ADI para mezcla de plaguicidas o para el total de aquellos que puedan consumirse por las vías dietética y no dietética.

Además de lo anterior, hay que destacar que la población del municipio también está expuesta a residuos de estas sustancias por el consumo de agua y alimentos contaminados. Como se mencionó anteriormente, se ha reportado la presencia de residuos de plaguicidas en rubros agrícolas producidos en esta región; específicamente, se encontraron concentraciones totales de 37 y $28 \mathrm{mg} / \mathrm{kg}$ en cáscara y pulpa de papa, respectivamente (Benítez-Díaz et al. 2015a) y alrededor de $47 \mathrm{mg} / \mathrm{kg}$ en fresas (Benítez-Díaz et al. 2015b). Por otra parte, trabajos realizados en diferentes acueductos de la zona mostraron que la concentración total de plaguicidas en agua potable alcanzaba a ser de $0.037 \mathrm{mg} / \mathrm{L}$ (Flores-García et al. 2011). Este conjunto de evidencias indica que el problema de exposición a residuos de plaguicidas en el municipio es multifactorial y multifuncional, y podría tener consecuencias importantes desde el punto de vista de la salud pública. En apoyo a esta observación, se han reportado problemas de salud atribuibles a la exposición a plaguicidas en trabajadores agrícolas y niños en edad escolar que habitan en la zona de estudio (Miranda-Contreras et al. 2013, 2015, 2017).

\section{CONCLUSIONES}

Se detectaron residuos de plaguicidas de diversos grupos químicos en suelos de uso agrícola de la microcuenca Los Zarzales, ubicada en el municipio Rivas Dávila del estado Mérida, Venezuela. En general, los niveles y tipos de principios activos varían de acuerdo con el estadio del cultivo, encontrándose la mayor concentración y variedad en la etapa media, para disminuir de manera importante en el tiempo de la cosecha. Se encontró una excepción a esta tendencia para el carbendazim, que se mantiene constante durante todo el periodo de producción, lo cual podría indicar que se aplica de manera excesiva. Por otra parte, la presencia de estos residuos podría constituir un potencial problema de exposición a una mezcla de sustancias tóxicas a través de la ingesta de polvo y suelo contaminados. Esta vía de exposición, sumada a otras previamente descritas en la literatura, podría contribuir a la generación de un problema de salud pública en la zona de estudio.

\section{AGRADECIMIENTOS}

Esta investigación fue financiada por el Fondo Nacional de Ciencia, Tecnología e Innovación (FONACIT) a través del proyecto S1-2002000281 y del Consejo de Desarrollo Científico, Humanístico, Tecnológico y Artes (CDCHTA) de la Universidad de Los Andes, Mérida, Venezuela, con la subvención CVI-ADG-M 08-95-03.

\section{REFERENCIAS}

Alam G. y Jones B. (2014). Toxicogenetics: In search of host susceptibility to environmental toxicants. Front. Genet. 5, 327. DOI: 10.3389/fgene.2014.00327 
Alister C., Araya M., Becerra K., Saavedra J. y Kogan M. (2017). Preharvest interval periods and their relation to fruit growth stages and pesticide formulations. Food Chem. 221, 548-554.

DOI: 10.1016/j.foodchem.2016.11.140

Benítez-Díaz P. y Miranda-Contreras L. (2009). Efectos de la exposición prenatal a paraquat sobre el desarrollo de la transmisión sináptica aminoacídica en la corteza cerebral parietal del ratón. Invest. Clin. 50 (4), 465-478.

Benítez-Díaz P. y Miranda-Contreras L. (2013). Contaminación de aguas superficiales por residuos de plaguicidas en Venezuela u otros países de Latinoamérica. Rev. Int. Contam. Ambie. 29 (número especial sobre plaguicidas), 7-23.

Benítez-Díaz P., Miranda-Contreras L., Molina-Morales Y., Sánchez-Gil B. y Balza-Quintero A. (2015a). Residuos de plaguicidas en la cáscara e interior de la papa (Solanum tuberosum L.) proveniente de una región agrícola del estado Mérida, Venezuela. Bioagro 27 (1), 27-36.

Benítez-Díaz P., Miranda-Contreras L., Balza-Quintero A., Sánchez-Gil B. y Molina-Morales Y. (2015b). Residuos de plaguicidas en fresa (Fragraria $x$ ananassa) cosechada en una región agrícola del estado Mérida, Venezuela. Bioagro 27 (3), 183-190.

Delgado F., Terrazas R. y López R. (1998). Planificación de la conservación de suelos en cuencas altas, utilizando relaciones erosión-productividad. Agronomía Trop. 48 (4), 395-411.

EUFIC (2013). Acceptable daily intakes (ADIs) [en línea]. European Food Information Council. http://www. eufic.org/en/understanding-science/article/qas-onacceptable-daily-intakes-adis 30/10/2017

EC (2016). EU pesticide database. European Commission [en línea]. http://ec.europa.eu/food/plant/pesticides/ eu-pesticides-database/public/?event=homepage\&la nguage $=$ EN 30/10/2017

FAO/OMS (2016). Codex Alimentarius: Normas internacionales de los alimentos. Ver las normas, aquí debe ir completo el nombre de las instituciones FAO y OMS http://www.fao.org/fao-who-codexalimentarius/home/ es/ 30/10/2017

Ferré D.M., Quero M., Hynes V., Saldeña E., Lentini V., Tornello M., Carracedo R. y Gorla N.B. (2018). Ensayo de micronúcleos de citoma bucal en trabajadores de fincas frutícolas que han aplicado plaguicidas alrededor de quince años. Rev. Int. Contam. Ambie. 34 (1): 2333. DOI: 10.20937/RICA.2018.34.01.02

FHIA (2007). Hoja técnica. Analicemos el suelo para alimentar adecuadamente los cultivos. Fundación Hondureña de Investigación Agrícola [en línea]. http://www. fhia.org.hn/dowloads/lab_quimico_agricola_pdfs/ hojatecnica1 analicelsuelo.pdf 30/10/2017
Flores-García M., Molina-Morales Y., Balza-Quintero A., Benítez-Díaz P. y Miranda-Contreras L. (2011). Residuos de plaguicidas en aguas para consumo humano en una comunidad agrícola del estado Mérida, Venezuela. Invest. Clin. 52 (4), 295-310.

García-Hernández J., Leyva-Morales J.B., Martínez-Rodríguez I.E., Hernández-Ochoa M.I., Aldana-Madrid M.L., Rojas-García A.E., Betancourt-Lozano M., Pérez-Herrera N.E. y Perera-Ríos J.H. (2018). Estado actual de la investigación sobre plaguicidas en México. Rev. Int. Contam. Ambie. 34 (número especial sobre contaminación y toxicología por plaguicidas [CTP]), 29-60. DOI: 10.20937/RICA.2018.34.esp01.03

Imfeld G. y Vuilleumier S. (2012). Measuring the effects of pesticides on bacterial communities in soil: A critical review. Eur. J. Soil Biol. 49, 22-30.

DOI: 10.1016/j.ejsobi.2011.11.010

Landaeta-Jiménez M., Aliaga C., Sifontes Y., Vásquez M., Ramírez G., Falque Madrid L., Herrera M., Reyes A.M., Elzakem E., Herrera H., Bernal J. y Marco E. (2013). Valores de referencia de energía para la población venezolana. Arch. Latinoam. Nutr. 63 (4), 258-277.

Mejías J. y Jerez J. (2006). Guía para la toma de muestras de residuos de plaguicidas aguas, sedimentos y suelos. Instituto de Investigaciones Agropecuarias, Chile [en línea]. http://www.sag.cl/sites/default/files/GUIA $\% 2$ 520TOMA\%2520MUESTRAS\%2520PLAGUICID AS.pdf 30/10/2017

Miranda-Contreras L., Gómez-Pérez R., Rojas G., Cruz I., Berrueta L., Salmen S., Colmenares M., Barreto S., Balza A., Zavala L., Morales Y., Molina Y., Valerie L., Contreras C.A. y Osuna J. (2013). Occupational exposure to organophosphate and carbamate pesticides affects sperm chromatin integrity and reproductive hormone levels among Venezuelan farm workers. J. Occup. Health 55 (3), 195-203.

DOI: $10.1539 /$ joh.12-0144-FS

Miranda-Contreras L., Cruz I., Osuna J., Gómez-Pérez R., Berrueta L., Salmen S., Colmenares M., Barreto S., Balza A., Morales Y., Zavala L., Labarca E., García N., Sanchez B., Contreras C.A. y Andrade H. (2015). Efectos de la exposición ocupacional a plaguicidas sobre la calidad del semen en trabajadores de una comunidad agrícola del estado Mérida, Venezuela. Invest. Clin. 56 (2), 123-136.

Miranda-Contreras L., Osuna J., Cruz I., Morales Y., Camacho N., Ciccheti R., Salazar I., Colmenares M., Balza A., Zavala L. y Sánchez B. (2017). Altered hematological and biochemical parameters in schoolchildren living in an agricultural community of Mérida State, Venezuela. J. Environ. Anal. Toxicol. 7, 431. DOI: 10.4172/2161-0525.1000431 
Molina-Morales Y., Flores-García M., Balza-Quintero A., Benítez-Díaz P. y Miranda-Contreras L. (2012). Niveles de plaguicidas en aguas superficiales de una región agrícola del estado Mérida, Venezuela, entre 2008 y 2010. Rev. Int. Contam. Ambie. 28 (4), 289301.

Morgan M., Wilson N. y Chuang J. (2014). Exposures of 129 preschool children to organochlorines, organophosphates, pyrethroids, and acid herbicides at their homes and daycares in North Carolina. Int. J. Environ. Res. Public Health. 11 (4), 3743-3764.

DOI: 10.3390/ijerph110403743

Peña-Contreras Z., Miranda-Contreras L., MoralesOvalles Y., Colmenares-Sulbarán L., Dávila-Vera D., Balza-Quintero A., Salem S. y Mendoza-Briceño R. (2016). Atrazine and mancozeb induce excitotoxicity and cytotoxicity in primary cultures of mouse cerebellar cortex. Toxicol. Environ. Chem. 98 (8), 958-976. DOI: $10.1080 / 02772248.2016 .1151020$

Richards J., Reid R., Luo Y. y Gan J. (2016). Distribution of pesticides in dust particles in urban environments. Environ. Pollut. 214, 290-298.

DOI: 10.1016/j.envpol.2016.04.025

Silvestre (2015). Protexín 500FW [en línea]. http:// www.silvestre.com.pe/site/images/Fichas_Tecnicas/ FT_PROTEXIN_500_FW_09.pdf 30/04/2019
Simcox N., Fenske R., Wolz S., Lee I. y Kalman D. (1995). Pesticides in household dust and soil: Exposure pathways for children of agricultural families. Environ. Health Perspect. 103 (12), 1126-1134.

DOI: $10.2307 / 3432609$

US-EPA (1996). Method 3540C, Soxhlet extraction. U.S. Environmental Protection Agency [en línea]. https://www.epa.gov/sites/production/files/2015-12/ documents/3540c.pdf 30/10/2017

US-EPA (2011). Soil and dust ingestion. En: Exposure factors handbook: 2011 edition. Office of Research and Development, National Center for Environmental Assessment, U.S. Environmental Protection Agency, Washington DC, EUA, pp. 5, 1-5, 52.

Uzcátegui J., Araujo Y. y Mendoza L. (2011). Residuos de plaguicidas organoclorados y su relación con parámetros físico-químicos en suelos del municipio Pueblo Llano, Estado Mérida. Bioagro 23 (2), 115-120.

Vymazal J. y Brezinová T. (2015). The use of constructed wetlands for removal of pesticides from agricultural runoff and drainage: A review. Environ. Int. 75, 11-20. DOI: 10.1016/j.envint.2014.10.026

Yu Y., Chu X., Pang G., Xian Y. y Fang H. (2009). Effects of repeated applications of fungicide carbendazim on its persistence and microbial community in soil. J. Environ. Sci. 21 (2), 179-185.

DOI: 10.1016/S1001-0742(08)62248-2 